\title{
24. PHOTOGRAPHIC ASTROMETRY (ASTROMETRIE PHOTOGRAPHIQUE)
}

PRESIDENT: C.A. Murray

VICE PRESIDENT: H.K. Eichhorn

ORGANIZING COMMITTEE: Ch. de Vegt, L.W. Fredrick, W. Gliese, R.S. Harrington, P. Lacroute, H.I. Potter, W.F. van Altena.

Although our Commission bears the title "Photographic Astrometry", we cannot ignore the development of modern techniques of observation and image detection. The long term prospects for astrometry are dominated by the plans for space astrometry now being considered by NASA and ESA.

A team consisting of van Altena (leader), Franz and Fredrick have completed their astrometric instrument definition study for the NASA Space Telescope. Preliminary designs have been developed for two instruments which are capable of measuring relative image positions with an accuracy of $\pm \$ 002$ for $m<17$. It is expected that parallaxes should be measurable to \pm !001. A proposal for astrometric use of the ST, with W.H. Jefferys (Texas) as principal investigator, has been submitted to NASA. In addition to scientific programmes, which include parallaxes, binaries and faint solar system astrometry, the responsibilities of the team include a basic service of guide star selection and acquisition for general use with ST.

A Phase A study of the ESA proposal for a dedicated astrometric satellite, to be known as Hipparcos, was. completed early in 1978; the science team for this study included Lacroute and Murray from this Commission. The study demonstrated the feasibility of Lacroute's original proposal to measure large angular distances by means of a two-field telescope. A possible programme would be to observe some $10^{5}$ stars during a 2-3 year mission; relative positions, annual proper motions and absolute parallaxes would be obtained with accuracy \pm "002 for $m<11$. The impact of both projects on astrophysics was the subject of a conference on European Satellite Astrometry which was held in Padova in June 1978.

IAU Colloquium No. 48 on "Modern Astrometry" was held in Vienna, in September 1978. Formally this was a colloquium of our Commission, but Commission 8 was also closely involved. A resolution was passed, urging the Presidents of both Commissions to set up a Joint Working Group to coordinate work on improving the stellar reference frame. A working party of radio and optical astrometrists was also set up with K.J. Johnston (NRL, Washington, DC) as chairman for coordinating the identification and precise position measurement of radio sources and their optical counterparts for improvement of the relationship between optical and radio reference frames.

In these fields of activity, the interests of our Commission coincide with those of Commission 8. It is important to optimise the available effort in setting up and maintaining the stellar reference frame, and maybe the time is ripe for a review of the responsibilities and even the titles, of our two commissions.

The study of nearby stars has always been a major interest for our commission. 
Plans are reported by van Altena at Yale, for a revision of the General Catalogue; these formed the subject of one formal session at Vienna, and also much discussion. Several papers have appeared recently, on systematic parallax errors and the use of parallaxes for absolute magnitude calibration (Turon Lacarrieu and Creze, Buchholz, Nфrgaard-Nielsen; 19-111-001, 005, 008). Lutz has extended his procedure for estimating bias in absolute magnitude calibration from parallaxes, to include the effect of a Gaussian luminosity function and apparent magnitude cut off. Hanson (19-111-006) has used observed proper motion distributions to improve the LutzKelker corrections, and has also found evidence for magnitude dependence in Allegheny parallaxes. Upgren, with his associates, has studied parallax errors (19-111-002) and has published an extensive review (20-111-001). Gliese reports plans for revision of his Catalogue of Nearby Stars. From data published since 1968 he has compiled three lists: (1) 293 stars with $\pi>0$ 040 which are not yet in the Catalogue; of these 181 have trigonometric parallax measured. (2) 163 suspects. (3) 374 new measurements of stars in the Catalogue, 68 of which now have $\pi<0 ! 045$

Other activities reported by Commission members are summarized in the following sections.

\section{Trigonometric Parallaxes - Nearby Stars}

Harrington reports that the US Naval Observatory is continuing its programme of measuring parallaxes of intrinsically faint stars, with the 6I-inch astrometric reflector at Flagstaff. At present there are about 200 stars on the programme. Fourth and fifth lists containing 93 and 95 stars respectively, have been published (Publ. US Nav. Obs. XXIV, pt. III (1976) and pt. IV (1978)).

Upgren reports the completion, during 1976-8, of twelve lists containing parallaxes of a total of about 250 stars determined at van Vleck Observatory; most of these are $K$ and $M$ dwarfs found by Vyssotsky.

In collaboration with J. Stock and D.J. MacConnell, Upgren has begun a parallax programme using the $65-\mathrm{cm}$ refractor of CIDA, the Venezuelan National Observatory. At a latitude of $8^{\circ} 48^{\prime} \mathrm{N}$, this telescope is well suited to the study of the systematic differences between northern and southern observatories. Stars for observation have been selected primarily from those already observed at Allegheny, McCormick, Yale or Cape, but other stars of astrophysical interest are being included. Coordination with programmes at van Vleck and Mt Stromlo (Ianna) is also planned.

At Sproul, parallaxes have been published for 98 stars from long-interval series; these are contained in 14 papers by van de Kamp, Lippincott, Heintz, Hershey and others. Long term behaviour of the Sproul refractor is being studied from data obtained since 1950. Experiments with larger numbers of reference stars and plate constants have shown minimal gain in accuracy.

Klemola has completed measurements for about 20 stars on the programme of the Lick 93-cm refractor; plates are now available for the remaining 80 stars of the programme, which was initiated by Vasilevskis.

The Yale parallax series has been resumed with the publication of results of 13 previously unmeasured series taken with the 26-inch refractor at Johannesburg and at Mt Stromlo (Astron. J. 83, p. 640, 1978). 
van Altena and L.H. Auer have completed an analysis of Yerkes parallax plates, measured on PDS microdensitometers and reduced with digital image centring methods. Average accuracy from 25 single exposure plates is \pm :004 (s.e.)

van Altena (Bul1. Am. Astron. Soc. 9, p. 599, 1978) reports results of a pilot programme using the prime focus of the Kitt Peak $4 \mathrm{~m}$ telescope. Observations to $B \sim 23$ have been completed in three mid-latitude fields. Preliminary measurements of one field give a parallax accuracy of \pm !0024 (s.e.) from 22 single exposure plates. About 4000 stars in each field will be measured.

At Pulkovo, Kanaev, A.A. Kiselev, V.A. Sokolova and others are continuing the measurement of parallaxes of bright stars, double stars, carbon stars, red dwarfs and some circumpolar stars, with the 26-inch refractor (18-111-006).

A second list of 26 stars measured at Herstmonceux has been published (B.G.F. Scales: Mon. Not. Roy. Astron. Soc. 184, p. 101) and results for a third list of 19 stars have been obtained; these are mostly $\mathrm{K}$ and $M$ dwarfs discovered by Vyssotsky.

Also at Herstmonceux, measurements have been made, with GALAXY, of about 15,000 images on each of more than 50 plates taken with the UK Schmidt $(1.2 \mathrm{~m})$ telescope, covering an area of $4 \frac{1}{2}^{\circ} \times 4 \frac{1}{2}^{\circ}$ near the South Galactic Cap. So far parallaxes have been obtained for 880 stars with $B<14$, with an external s.e. of \pm !018. (Murray and Corben, Mon. Not. Roy. Astron. Soc. in press).

K. Kamper (David Dunlap Observatory) and Wesselink (Yale) have completed their study of the $\alpha$ - Proxima Centauri System from plates taken with the Yale and Cape refractors, and the double astrograph at El Leoncito. They find that the space motion of $A B$ and Proxima are equal in size and direction, but point out that the orbital motion of Proxima around $A B$ should be detectable within a century.

Deutsch has found perturbations with 6 and 12 year periods in the motion of 61 Cygni from Pulkovo and Sproul data, and estimates the mass causing the 6 year perturbation to be 4J. (19-118-001; Pis'ma v. Astron. Zh. 4, P. 95, 1978).

\section{Proper Motions and Kinematics}

Luyten has published a new catalogue of some 3600 stars with annual proper motion exceeding $0 ! 5$, and estimates that the total number of such stars with $m_{p g}$ $<21$ in the whole sky is about 4350. Unpublished machine-readable data for more than $2 \times 10^{5}$ stars with annual proper motion between $0: 18$ and 0 !'09, found with the automated-computerized blink machine, have been deposited with the NASA Data Center at Greenbe1t, Maryland. A new catalogue (NLLT) which is expected to contain data for about 60,000 stars with annual proper motion exceeding $0 ! 18$ will be in press before the next General Assembly.

An analysis of the reduced proper motions and colours of 115,000 stars was presented by Luyten at the Russell Symposium (1977); this shows clearly that nearby $M$-dwarfs with small proper motion are not as abundant as has been postulated recently.

Klemola reports that by the end of 1978, second epoch plate material will have been obtained for nearly 900 of the 1246 Lick fields. Measurements have been completed for about 450 fields with $-3^{\circ}<\delta<43^{\circ}$ and outside the zone of avoidance; 
final reductions are in progress. Faint stars are used for plate constants, and galaxies give the reduction to absolute proper motion. Representative samples of stars from McCormick fields and the KSZ programme are also being measured.

The Yale Southern Observatory, in collaboration with Felix Aguilar Observatory of the University of San Juan is extending the region of overlap with the Lick programme; a partial overlap for $-23^{\circ}<\delta<28^{\circ}$ will be obtained.

In USSR work continues at Pulkovo, Kiev and the Sternberg Astronomical Institute on the KSZ programme of measuring proper motions relative to galaxies. At Tashkent, A.G. Rakhimov has published 5 catalogues of proper motions in areas of the KSZ programme (20-112-011, 012, 013, 014, 016) and at Kiev, L.P. Panteleeva has studied the quality of galaxy images obtained with various astrographs (Soobsch. Gos. Astr. Inst. Stemberg, No. 193, p. 14, 1977). Investigations of magnitude differences between various proper motion catalogues by Rakhimov (20-112-010; Tsirk. Astr. Inst. Tashkent, No. 60, p. 9, 1976) and S.P. Rybka (19-112-007; Astrometrija i Astrofizika 35, p. 3, 1978) indicate a possible cause for divergence in the measurement of precession.

Quijano reports that at San Femando (Cadiz) second epoch photography of 48 KSZ areas will start in 1979 .

Work on star clusters at Yale, and at Hamburg where absolute proper motions of 40 clusters are being measured, has been reported to Commission 37 . Also at Hamburg, U.K. Gehlich is studying Perseus OB2. While at Yale, Hanson redetermined the Hyades distance modulus ( $3.30 \pm 0.06$ ) from Lick proper motions. At Pulkovo, V.N. Frolov (20-153-035) has studied four clusters in Cassiopeia, and at Tashkent, Latypov has published proper motions in NGC 6709 and 6940 (20-112-015, 017). A.I. Yatsenko has measured motions of trapezium type systems, (Astrometriji $i$ Astrofizika 55, p. 9). At Sydney, Robertson has published proper motions in NGC 6025 (20-112-019), and D. King in NGC 3532 ( $J 1$. Roy. Soc. NSW 111 , p. I); King is now studying NGC 2516 and 4103.

Researches on kinematics include an analysis of AGK3 and SAO (South) proper motions by Dieckvoss for solar motion and galactic rotation (Astron. Astrophys. 62, p. 445) and velocity ellipsoids; investigation of space motions is continuing. B.F. Jones and Klemola have determined proper motions for 210 Sanduleak stars near the North Galactic Pole, and have measured a sample of faint blue stars in a field at $8^{\mathrm{h}} 48^{\mathrm{m}}$, $+18^{\circ}$ on Palomar Schmidt plates at the suggestion of Sandage. Jones continues his work on $\mathrm{T}$ Tau and other stars in the Taurus dark cloud. At Tokyo, T. Mikami has determined statistical parallaxes of $F, G, K, M$ type stars using AGK3 proper motions and various sources for radial velocities (Pub. Astron. Soc. Japan, 30, pp. 191 and 209) and has also compiled data for 1490 M-type and 321 carbon stars (Ann. Tokyo. Astron. Obs. 17, No. 1).

\section{Photographic Positions and Catalogues}

Optical positions of radio sources are being determined at US Naval observatory, including $15-\mathrm{cm}$ transit circle and $20-\mathrm{cm}$ twin astrograph observations.

Positions of 48 sources have been measured jointly at Cambridge (Schmidt) and Herstmonceux (Refractors, Isaac Newton Telescope); the results will be published in the proceedings of the Vienna Colloquium.

Klemola has compiled a catalogue of 5000 reference stars for JPL to be used 
for alignment of cameras on the Voyager spacecraft, which will pass Jupiter in 1979.

Quijano reports that the Carte-du-Ciel astrograph at San Fernando (Cadiz) is engaged in observing 20 selected minor planets for the Leningrad ITA programme.

Lacroute and Velbousquet have completed the re-reduction of the Carte-du-Ciel plates between $-2^{\circ}<\delta<31^{\circ}$, using the Strasbourg AGK2/3 as reference data (20-002009).

Eichhorn and Hoffleit have continued work on the Yale $-60^{\circ}$ to $-70^{\circ}$ zone.

Nicholson reports that measurement of the Cape overlap survey plates has begun on the GALAXY machine at Herstmonceux; so far about 100 plates $\left(\delta=-44^{\circ}\right)$ have been measured.

Work with the Sydney astrograph has been reported to Commission 8 .

In the USSR, a founfold overlap survey of the Northem Hemisphere by several observatories is planned.

\section{Instrumentation and Measurement}

The El Leoncito double astrograph was re-collimated by R. Platzeck (La Plata) and van Altena; new tailpiece assemblies, plateholders and an image dissector auto-guider are being installed.

A new 400/2000 Zeiss (Jena) astrograph has been installed at Goloseevo, (I.G. Kolchinskij, C.P. Major; Vestnik Akademii Nauk Ukr. SSR, 1978, 4 ).

A $400 \mathrm{~mm}$ astrograph has been constructed at Engelhardt Observatory, for observations of planets and the Moon (Sh. T. Khabibulin; N.G. Rizvanov; Izv. Engelhardt Astron. Obs. 1976. No. 41-42, p. 104).

Performance of the $400 / 2000$ astrograph of the Zvenigorod Observatory has been studied by Muhammed Oman Sulejman. (Nauchn. Inf. 1978. No. 39).

Pilowski has proposed a new method of measuring the relative positions of galaxy and AGK3 star images on Schmidt plates, using narrow band filters for magnitude and colour compensation, with the coated layer removed from the central regions over the galaxy image.

P. Connes has put forward a comprehensive proposal for improving the accuracy of ground based parallax measurements, including a special helium filled telescope (Eso Conference. Optical Telescopes of the future). (Cadiz).

A new Zeiss ASCORECORD measuring machine has been installed at San Fernando

\section{Reduction Methods}

A.A. Kiselev and S.M. Chantariya have developed an algorithm for determining 
proper motions on the AGK3 system, by combining Carte-du-Ciel and modern photographic positions, which they have applied to the region of $\alpha$ Per (19-112-011).

A geometrical method of plate reduction has been developed by Kiselev, $0 . v$. Kiyaeva and $0 . A$. Kalinichenko, and applied in the investigation of distortion in wide-angle cameras (19-031-231).

Yu. F Zinchenko and Podobed have described a modification of the overlapping plate method to be used for the polar catalogue (20-041-055).

Fimeis reports on a statistical investigation of astrometric positions by means of the parameter and error covariance matrices; theoretical formulae have been tested by Monte Carlo methods.

Dommanget has adapted his programmes for the reduction of comet and asteroid positions, for use with star catalogues stored on disc. He has also developed his new method of reduction of astrometric plates, which will be applied in fields containing open clusters.

\section{Acknowledgements}

I am very grateful to all who responded to my request for information on which this report is based.

C. A. MURRAY

President of the Commission 